\title{
The correlation of monocyte to high-density lipoprotein ratio with uric acid and possible atherosclerotic pathway in patients treated with isotretinoin
}

\author{
Nurcan Metin ${ }^{1}$ and Cagri Turan ${ }^{2}$ \\ ${ }^{1}$ Affiliation not available \\ ${ }^{2}$ Erzurum Training and Research Hospital
}

October 5, 2020

\begin{abstract}
Purpose: We aimed to reveal the relationship of uric acid with MHR and other inflammatory markers acne patients before and after isotretinoin treatment. In this way, we can try to shed light on the relationship between isotretinoin treatment and atherosclerosis. Methods: Two hundred twenty-four acne patients who administered isotretinoin (0.5-1 mg/ $\mathrm{kg} / \mathrm{day}) \mathrm{were}$ enrolled in the study. In the pre-treatment phase and 3 months after treatment, MHR, SUA, mean platelet volume, plateletcrit, neutrophil-lymphocyte ratio (NLR), platelet-lymphocyte ratio, monocyte-lymphocyte ratio, serum triglyceride, total cholesterol, high-density lipoprotein (HDL), and low-density lipoprotein (LDL) levels of the patients were analyzed. Results: Compared to the pre-treatment phase, three months after treatment, there was a statistically decrease in neutrophil count and an increase in lymphocyte count (p: 0.002, p: 0.011; respectively). Accordingly, there was a statistically significant decrease in NLR (p: 0.001). It was noteworthy that MHR and SUA levels increased significantly (p: 0.042, p: 0.010; respectively) and there was a positive correlation between SUA level and MHR (r: 0.212, p: 0.012). Serum total cholesterol, LDL, and triglyceride levels increased and HDL levels decreased significantly after treatment (p: 0,001). Conclusion: This study contributes to the comprehension of the relationship between isotretinoin treatment and atherosclerosis, which has been frequently reported in the literature. It was thought that the isotretinoin-induced uric acid increase might be related to dyslipidemia. Isotretinoin may initiate the atherosclerotic process in vascular endothelial and smooth muscles, with uric acid increase and HDL decrease. An increase in MHR is also an inflammatory marker indicating this process.
\end{abstract}

\section{Introduction}

Acne is a common chronic inflammatory disease of the pilosebaceous unit. Four key factors play a role in pathogenesis with interrelated mechanisms, such as increased sebum production, hyperkeratinization of the follicular infundibulum, inflammation, and Cutibacterium acnes proliferation. Primary lesion types in acne are comedones and inflammatory lesions (papules, pustules, and nodules). The typical distribution of acnes includes the face, upper back, chest, and shoulders rich in sebaceous glands. The treatment approach can vary depending on the type, distribution, severity, and degree of effect on the patient. ${ }^{1}$

Isotretinoin (ISO) is a synthetic analogue of vitamin $\mathrm{A}^{2}$. ISO treatment is effective in all four steps of acne pathogenesis. ${ }^{2}$ However, it has numerous side effects related to many systems such as hepatic, renal, musculoskeletal, hematological, neuropsychiatric, and gastrointestinal systems. Also, some conflicting publications have been reported on its relationship with some inflammatory diseases such as inflammatory bowel disease and sacroiliitis. ${ }^{2-4}$ In clinical practice, patients are followed up with laboratory tests such as complete blood count $(\mathrm{CBC})$, liver and kidney function tests, and lipid profile. 
Mean platelet volume (MPV) and plateletcrit (PTC) indicate the platelet function and percentage of platelet volume in the blood; respectively. MPV, PTC, neutrophil-lymphocyte ratio (NLR), and platelet-lymphocyte ratio (PLR) were used as inflammatory markers in various studies for many diseases and drugs, including ISO treatment. ${ }^{5-7}$ Monocyte to high-density lipoprotein ratio (MHR) is a recently defined inflammatory biomarker associated with metabolic syndrome and atherosclerosis. ${ }^{6,8}$ Separate studies have reported an increase in MHR and carotid intima-media thickness (CIMT) in acne patients after ISO treatment. ${ }^{9,10}$ Although ISO is known to predispose to hyperuricemia, there is not enough clear information in the literature about the mechanism of triggering the increase in serum uric acid (SUA) level. ${ }^{11}$ Lately, some studies emphasize that hyperuricemia is a risk factor for both metabolic syndrome and cardiovascular diseases (CVD), which is also associated with values such as MHR and PLR. ${ }^{6,12,13}$ In a recent study consisting of more than 8000 participants was reported for the first time in the literature that MHR has a positive correlation with SUA and that it can be used in predicting the risk stratification of hyperuricemia. ${ }^{14}$

In this study, we aimed to reveal the relationship of uric acid with MHR and other inflammatory markers stated above, in moderate-severe acne patients before and after ISO treatment. In this way, we can try to shed light on the frequently reported relationship between ISO treatment and atherosclerosis.

\section{Materials and Methods}

Between 1 June- 31 December 2019, 224 patients with moderate-severe acne vulgaris who administered oral ISO $(0.5-1 \mathrm{mg} / \mathrm{kg} /$ day) at the Department of Dermatology and Venereology of the Regional Training and Research Hospital were included in this study. Written consent forms were obtained from 203 adult patients and parents of 21 adolescent patients. Patients who used any medication (iron, vitamin supplements, statins, etc.) that could affect hematological parameters and lipid profile in the last 3 months were excluded. All patients were selected from the group of 15-35 years of age to be able to minimize the possibility of atherosclerosis comorbidity as it may affect the inflammatory parameters. Patients with cancer, severe infection, hematological diseases (including iron-deficiency anemia), heart failure, and lung diseases were excluded. Data such as gender, age, CBC, lipid profile, and the serum alanine aminotransferase (ALT), aspartate aminotransferase (AST), blood urea nitrogen (BUN), creatinine, and uric acid levels were obtained from the electronic registration database. In the pre-treatment phase and 3 months after treatment, white blood cell (WBC), neutrophil, lymphocyte, monocyte and platelet counts, MPV, PTC, NLR, PLR, monocyte/lymphocyte ratio (MLR), MHR, serum triglyceride (TG), total cholesterol, high-density lipoprotein (HDL), and low-density lipoprotein (LDL) levels were analyzed.

This single-center cross-sectional retrospective-study was approved by the Ethics Committee of the Regional Training and Research Hospital, Erzurum, Turkey (Decision No: 2020/12-134). This study was conducted per the latest version of the "Helsinki Declaration" and "Guidelines for Good Clinical Practice".

\section{Laboratory measurements}

Blood samples of the patients were taken in ethylenediaminetetraacetic acid (EDTA) tubes and CBC values, including the platelet, lymphocyte, neutrophil, and monocyte counts, and MPV were measured using an automated blood counting system (Beckman Coulter LH 780, Brea, California, USA).

\section{Statistical analysis}

All data were analyzed using the IBM Statistical Package for the Social Sciences (SPSS) version 21.0 and MS-Excel 2010 software. The normality distribution of scale variables was checked using the KolmogorovSmirnov test. Data were expressed as median (interquartile range) or mean \pm standard deviation for those with non-parametric or parametric distribution, respectively. The paired sample t-test or Wilcoxon test was used for dependent samples, whichever is appropriate. Independent samples were compared with the MannWhitney U test. Pearson's chi-square test was used for categorical variables. Scale data were compared with 
Pearson's or Spearman's correlation tests according to their distributions. Two-sided p-values less than 0.05 were considered statistically significant.

\section{Results}

Of the 244 acne patients participating in the study, 173 were women $(77.2 \%)$. The mean age of the patients was $21.0 \pm 3.5$ years. There was no difference between genders in terms of age ( $p: 0.263)$. The changes in laboratory parameters in the $3^{\text {rd }}$ month of ISO treatment compared to the $0^{\text {th }}$ month are presented in Table 1. After treatment, there was a statistically decrease in neutrophil count and an increase in lymphocyte count (p: 0.002, p: 0.011; respectively). Accordingly, there was a statistically significant decrease in NLR (p: 0.001). A statistically significant increase in total cholesterol, LDL, triglyceride levels, and a significant decrease in HDL levels were found $(\mathrm{p}<0.001)$. It was noteworthy that MHR and SUA levels increased significantly (p: 0.042, p: 0.010; respectively). Correlations of SUA levels with laboratory parameters before and after treatment were presented in Table 2. SUA and hemoglobin levels were moderately positively correlated (r: $0.439, \mathrm{p}<0.001$ and $\mathrm{r}: 0.473, \mathrm{p}<0.001$; respectively). Although there was no significant relationship between SUA level with total cholesterol, LDL, and triglyceride levels before treatment, a weakly significant relationship was found between them after treatment (Table 2). The correlation between MHR and SUA levels before and after treatment was statistically significant (r: 0.157, p: 0.048; r: 0.212, p: 0.012; respectively).

\section{Discussion}

Chiang et al. stated that hyperuricemia could be a sign of a metabolic disorder that is a precursor of hypertension, hypertriglyceridemia and diabetes mellitus, and high SUA levels may play a role in CVD development. ${ }^{15}$ Kang et al. emphasized that hyperuricemia can induce CRP mRNA expression in vascular endothelium and smooth muscle cells and reduce nitric oxide (NO) release. ${ }^{16}$ In another study of a murine model with metabolic syndrome, hyperuricemia was reported to cause a proinflammatory endocrine imbalance in adipose tissue. ${ }^{17}$ Although the association of atherogenic dyslipidemia (high TG and LDL and low HDL levels) and hyperuricemia was reported in many other studies, the physiopathology of this association has not been fully elucidated yet. ${ }^{18,19}$ Inconsistent with the previous studies, we have found that there was a statistically significant increase in SUA levels in the $3^{\text {rd }}$ month of ISO treatment. We have also found higher SUA levels in patients with atherogenic dyslipidemia.

Various studies reported that ISO treatment could increase the inflammatory response and oxidative stress. $^{20,21}$ Among the inflammation biomarkers, we have found that only MHR values significantly increased. MPV, PLR, and PCT values did not change, and NLR values decreased significantly. We have found no correlation between hyperuricemia and these inflammation biomarkers other than MHR. There are contradictory studies in the literature about the effects of ISO on CBC test parameters. ${ }^{10,22,23}$ In contrast to the study of Önder et al., we have found a significant decrease in NLR values in the $3^{\text {rd }}$ month of ISO treatment, similar to the study of Duman et al. ${ }^{10,23}$ ISO was reported to have an anti-inflammatory effect on patients with acne vulgaris by suppressing the chemotaxis of WBCs. ${ }^{24}$ It was thought that the decrease in NLR values indicated the anti-inflammatory effect of ISO.

Recent clinical and epidemiological studies have shown that MHR could predict various inflammatory diseases such as metabolic syndrome, diabetes mellitus, CVD, and acne vulgaris. ${ }^{8,10,13,25,26}$ Similarly, we have found a statistically significant increase in MHR values in the $3^{\text {rd }}$ month of ISO treatment. Monocytes interact with the damaged endothelium and cause gene overexpression of proinflammatory cytokines and adhesion molecules. On the other hand, HDL-cholesterol has anti-inflammatory, antioxidant, and vasorelaxant effects. ${ }^{27,28}$ The relationships between MHR and SUA with inflammatory diseases are similar. ${ }^{15}$ We have found that MHR values and SUA levels showed a weakly positive correlation both before and after ISO treatment. As far as we know, the relationship between MHR and SUA has not been reported in patients 
receiving ISO treatment to date. Both of these parameters are associated with atherosclerosis and metabolic syndrome. It was found that CIMT increases after 4 months in acne patients receiving ISO treatment, so it has been suggested that long-term ISO use increased atherosclerosis risk. ${ }^{9}$ In another study, it was claimed that ISO triggered the development of subclinical atherosclerosis in men. ${ }^{29}$ In the light of all these data and the findings of our study, we can explain the atherosclerosis pathway seen in Figure 1. It was thought that the ISO-induced uric acid increase might be related to dyslipidemia. ISO may initiate the atherosclerotic process in vascular endothelial and smooth muscles, with uric acid increase and HDL decrease. An increase in MHR value is also an inflammatory marker indicating this process.

The limitations of our study were that the study was retrospective, and other commonly used systemic inflammatory markers such as high sensitive C-reactive protein, erythrocyte sedimentation rate, and ddimer were not evaluated. We could not create a "healthy control group" because our study was retrospective and a comprehensive study reported that there was already a relationship between SUA and MHR.

In conclusion, this study contributes to the comprehension of the relationship between ISO treatment and atherosclerosis, which has been frequently reported in the literature. However, our study is insufficient to establish a cause-result link. More comprehensive studies are needed.

\section{References}

1. Zaenglein AL. Acne vulgaris. N Engl J Med 2018;379(14):1343-1352.

2. Brzezinski P, Borowska K, Chiriac A, Smigielski J. Adverse effects of isotretinoin: A large, retrospective review. Dermatol Ther 2017;30(4):e12483.

3. Crockett SD, Porter CQ, Martin CF, Sandler RS, Kappelman MD. Isotretinoin use and the risk of Inflammatory Bowel Disease: A case control study. Am J Gastroenterol 2010;105(9):1986.

4. Baykal Selçuk L, Aksu Arıca D, Baykal Şahin H, Yaylı S, Bahadır S. The prevalence of sacroiliitis in patients with acne vulgaris using isotretinoin. Cutan Ocul Toxicol 2017;36(2):176-179.

5. Ataseven A, Kurtipek GS, Ozturk P. Neutrophil lymphocyte ratio in patients receiving isotretinoin for acne vulgaris. Med-Science 2014;3:1026-1031.

6. Haybar H, Pezeshki SMS, Saki N. Evaluation of complete blood count parameters in cardiovascular diseases: An early indicator of prognosis? Exp Mol Pathol 2019:104267.

7. Tezcan D, Turan Ç, Yılmaz S, et al. What do simple hematological parameters tell us in patients with systemic sclerosis? Acta Dermatovenerol Alp Pannonica Adriat 2020;29(3):101-107.

8. Uslu AU, Sekin Y, Tarhan G, Canakcı N, Gunduz M, Karagulle M. Evaluation of monocyte to highdensity lipoprotein cholesterol ratio in the presence and severity of metabolic syndrome. Clin Appl Thromb Hemost 2018;24(5):828-833.

9. Saklamaz A, Uyar B, Yalcin M, Cengiz H. Isotretinoin increased carotid intima-media thickness in acne patients. Hippokratia 2016;20(1):14.

10. Önder S, Ozturk M. Can monocyte/HDL show inflammatory activity of isotretinoin treatment in acne patients? Cutan Ocul Toxicol 2020;39(2):111-114.

11. Solak B, Erdem T, Solak Y. Isotretinoin use for acne vulgaris is associated with increased serum uric acid levels. J Dermatolog Treat 2017;28(1):82-85.

12. Bando H. Recent Topics of Hyperuricemia for Metabolic Syndrome, Cardiovascular, and Chronic Renal Diseases. J Diab Obes Metab 2020;3(1):e104.

13. Chen JW, Li C, Liu ZH, et al. The role of monocyte to high-density lipoprotein cholesterol ratio in prediction of carotid intima-media thickness in patients with type 2 diabetes. Front Endocrinol (Lausanne) 2019;10:191. 
14. Chen M-Q, Shi W-R, Shi C-N, Zhou Y-P, Sun Y-X. Impact of monocyte to high-density lipoprotein ratio on prevalent hyperuricemia: findings from a rural Chinese population. Lipids Health Dis 2020;19(1):1-9.

15. Chiang K-M, Tsay Y-C, Vincent Ng T-C, et al. Is hyperuricemia, an early-onset metabolic disorder, causally associated with cardiovascular disease events in han Chinese? J Clin Med 2019;8(8):1202.

16. Kang D-H, Park S-K, Lee I-K, Johnson RJ. Uric acid-induced C-reactive protein expression: implication on cell proliferation and nitric oxide production of human vascular cells. J. Am. Soc. Nephrol. 2005;16(12):3553-3562.

17. Baldwin W, McRae S, Marek G, et al. Hyperuricemia as a mediator of the proinflammatory endocrine imbalance in the adipose tissue in a murine model of the metabolic syndrome. Diabetes 2011;60(4):1258-1269.

18. Ali N, Rahman S, Islam S, et al. The relationship between serum uric acid and lipid profile in Bangladeshi adults. BMC Cardiovasc Disord 2019;19(1):42.

19. Peng T-C, Wang C-C, Kao T-W, et al. Relationship between hyperuricemia and lipid profiles in US adults. Biomed Res Int 2015;2015.

20. Georgala S, Papassotiriou I, Georgala C, Demetriou E, Schulpis KH. Isotretinoin therapy induces DNA oxidative damage. Clin Chem Lab Med 2005;43(11):1178-1182.

21. Ozkol HU, Ozkol H, Karadag AS, Bilgili SG, Tuluce Y, Calka O. Oral isotretinoin therapy of acne patients decreases serum paraoxonase-1 activity through increasing oxidative stress. Drug Chem Toxicol 2015;38(1):63-66.

22. Tamer F, Yüksel ME, Avcı E. Is mean platelet volume an inflammatory marker in acne patients treated with isotretinoin? Acta Dermatovenerol Alp Pannonica Adriat 2019;28(2):65-69.

23. Duman N, Uzunali E, Manav V. A comparative study on hematological inflammation markers in Acne Vulgaris. IBRR 2015:1-5.

24. Karadag A, Ertugrul D, Bilgili S, Takci Z, Akin K, Calka O. Immunoregulatory effects of isotretinoin in patients with acne. Br J Dermatol 2012;167(2):433-435.

25. Satilmis S. Role of the monocyte-to-high-density lipoprotein ratio in predicting atrial high-rate episodes detected by cardiac implantable electronic devices. North Clin Istanb 2018;5(2):96.

26. Turkmen D, Altunisik N, Sener S. Investigation of monocyte HDL ratio as an indicator of inflammation and complete blood count parameters in patients with acne vulgaris. Int J Clin Pract 2020:e13639.

27. Thacker SG, Zarzour A, Chen Y, et al. High-density lipoprotein reduces inflammation from cholesterol crystals by inhibiting inflammasome activation. Immunology 2016;149(3):306-319.

28. Mestas J, Ley K. Monocyte-endothelial cell interactions in the development of atherosclerosis. Trends Cardiovasc Med 2008;18(6):228-232.

29. Karapınar T, Polat M, Buğdaycı G. Evaluation of subclinical atherosclerosis in Turkish patients with acne vulgaris receiving systemic isotretinoin. Dermatol Ther 2020;33(3):e13307.

\section{Tables}

Table 1. Change in blood parameters compared to $0^{\text {th }}$ month in the $3^{\text {rd }}$ month of ISO treatment

\section{Parameters}

Hemoglobin $(\mathrm{g} / \mathrm{dl})$

White blood cell $\left(10^{6} / \mathrm{l}\right)$ 
Neutrophil $\left(10^{6} / 1\right)$

Lymphocyte $\left(10^{6} / 1\right)$

Monocytes $\left(10^{6} / \mathrm{l}\right)$

Platelet $\left(10^{9} / \mathrm{l}\right)$

$\operatorname{PCT}\left(10^{-2}\right)$

$\operatorname{MPV}(\mathrm{f})$

NLR $\left(10^{-2}\right)$

$\operatorname{MLR}\left(10^{-2}\right)$

PLR

Total cholesterol $(\mathrm{mg} / \mathrm{dl})$

HDL (mg/dl)

LDL $(\mathrm{mg} / \mathrm{dl})$

Triglyceride $(\mathrm{mg} / \mathrm{dl})$

$\operatorname{MHR}\left(10^{5} / \mathrm{mg}\right)$

$\operatorname{ALT}(\mathrm{U} / \mathrm{l})$

$\operatorname{AST}(\mathrm{U} / \mathrm{l})$

BUN (mg/dl)

Creatinine $(\mathrm{mg} / \mathrm{dl})$

Uric acid $(\mathrm{mg} / \mathrm{dl})$

Data are expressed as median (interquartile range) or mean \pm standard deviation for those with non-parametric or parame

Table 2. Correlation of blood parameters with SUA levels at $0^{\text {th }}$ and $3^{\text {rd }}$ months

\section{Parameters}

Hemoglobin (g/dl)

White blood cell $\left(10^{6} / 1\right)$

Neutrophil $\left(10^{6} / 1\right)$

Lymphocyte $\left(10^{6} / 1\right)$

Monocytes $\left(10^{6} / 1\right)$

Platelet $\left(10^{9} / \mathrm{l}\right)$

$\operatorname{PCT}\left(10^{-2}\right)$

$\operatorname{MPV}(\mathrm{fl})$

$\operatorname{NLR}\left(10^{-2}\right)$

$\operatorname{MLR}\left(10^{-2}\right)$

PLR

Total cholesterol $(\mathrm{mg} / \mathrm{dl})$

HDL (mg/dl)

LDL (mg/dl)

Triglyceride $(\mathrm{mg} / \mathrm{dl})$

$\operatorname{MHR}\left(10^{5} / \mathrm{mg}\right)$

BUN (mg/dl)

Creatinine (mg/dl)

Pearson's correlation was used. Spearman correlation was used for all others. Significant values were shown in bold. PCT:

\section{Figures}

Figure Legend

Figure 1. Possible atherosclerotic pathway induced by isotretinoin 


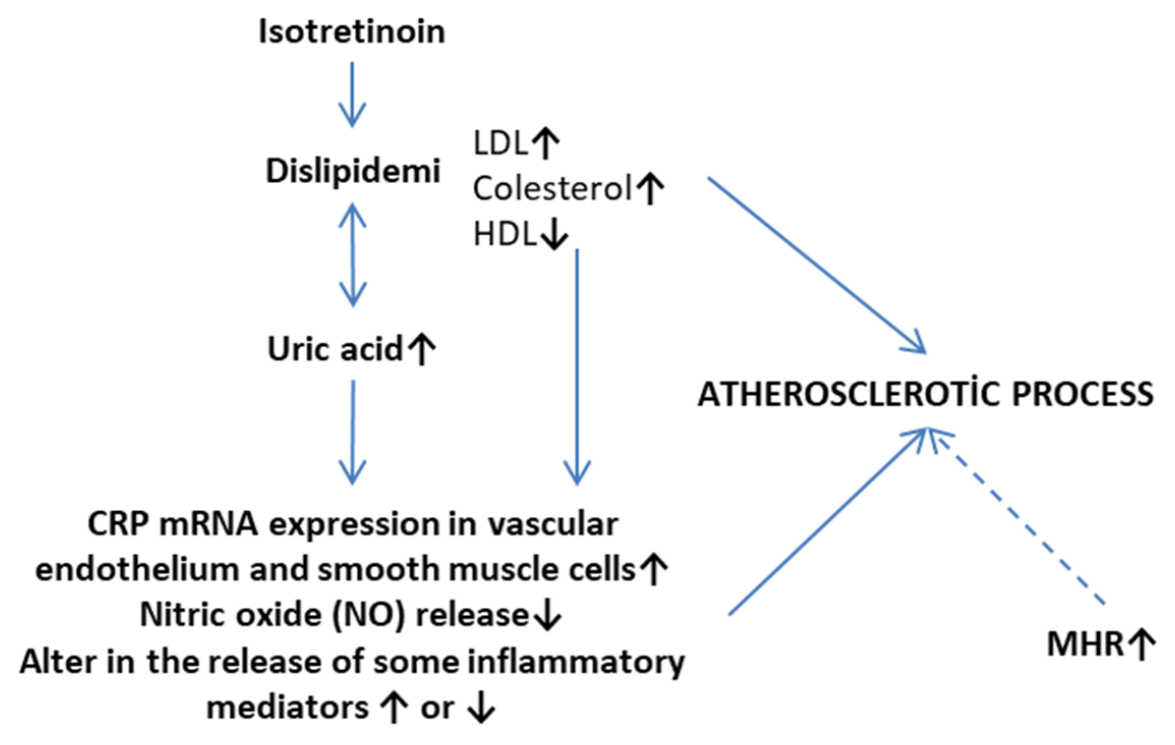

